\title{
Design of A MIMO OFDM Baseband Transceiver for Cognitive Radio System
}

\author{
Jui-Ping Lien, Po-An Chen and Tzi-Dar Chiueh \\ Graduate Institute of Electronics Engineering and \\ Department of Electrical Engineering, National Taiwan University, Taipei, Taiwan \\ Email: pa@analog.ee.ntu.edu.tw
}

\begin{abstract}
As wireless communication services become more prevalent, bandwidth requirement increases dramatically. As such, the concept of Cognitive Radio (CR) has recently received much attention. In this paper, we present a MIMO-OFDM based CR system capable of dynamically adjusting its system parameters according to spectrum allocation. From system simulation, the proposed transceiver is shown to be a promising solution for CR applications.
\end{abstract}

\section{INTRODUCTION}

Recently, wireless communication is becoming pervasive. The available spectrum, however, is getting scarce and the conventional fixed spectrum allocation and usage has now become inefficient [1]. Cognitive Radio (CR) is proposed and widely discussed to solve this dilemma [2]. The basic idea of CR is to provide a system with the ability to sense available spectrum slots not occupied by existing users and detect whether any primary user is demanding the bands that the CR system currently uses. In 2003, a regulation enacted by FCC formally initialized the commercialization of CR [3]. Currently, CR plays a major role in many applications to enhance communication performance and/or provide advanced services [4]. Since CR only uses non-contiguous bands in the spectrum, Orthogonal Frequency Division Multiplexing (OFDM) is considered a suitable transmission technique. In brief, the primary target of CR is to find out available bands and change, if necessary, system parameters such as carrier frequency, transmission bandwidth, power consumption and modulation type to achieve efficient use of spectrum resource.

In this paper, a MIMO-OFDM CR system for $5 \mathrm{GHz}$ UNII band to coexist with IEEE 802.11a Primary Users (PU) is proposed. The proposed system can sense the channel characteristics to actively modify its physical layer settings through parameterized design so that existing PU are not interfered by the CR signals.

The rest of the paper is organized as follows. In Section 2, the system specification and channel model will be introduced. The design of the transceiver is discussed in Section 3. In Section 4, simulation of the CR system will be given. Finally, Section 5 concludes this paper.

\section{System Specification And Channel Model}

There are two basic considerations in the overall physical layer specification design: dynamic spectrum allocation and channel characteristic. As the primary users change their use of spectrum, the CR system needs to adapt its bandwidth, carrier frequency and spectrum allocation to avoid system malfunction. In the proposed CR system, the operating frequency is $5 \mathrm{GHz}$ UNII band, the same as that of IEEE $802.11 \mathrm{a}$ wireless system. The targeted $5 \mathrm{GHz}$ UNII band can be divided into lower band, $5.15-5.35 \mathrm{GHz}$, and upper band, $5.725-5.825 \mathrm{GHz}$, and our system will use either one of them according to the overall system requirements. Based on the above system requirements and OFDM design considerations, we define the overall system specifications.

\section{A. System Specification}

Summary of the major specification is shown in Table I, manifesting the flexibility of the proposed system. The basic parameter setting is from that of IEEE $802.11 \mathrm{a}$, bearing the OFDM symbol duration of $4-\mu s$, resulting from the sum of FFT and guard interval (GI) period. Since one subband is $20 \mathrm{MHz}$ and the possible number of subband allocation is $1,2,4$ or 8 , we can get the corresponding bandwidths. As sampling rate is the same as bandwidth, different FFT lengths can be obtained. The number of subcarrier used is less than the corresponding FFT lengths since there are guard bands to avoid out-of-band interference. Finally, to provide multiple data rate, V-BLAST 2 × 2 MIMO scheme is implemented [5].

TABLE I

SYSTEM SPECIFICATIONS

\begin{tabular}{|c|c|c|}
\hline & Lower band & Upper band \\
\hline Frequency band(GHz) & $5.15-5.35$ & $5.725-5.825$ \\
\hline Subband Number & 8 & 4 \\
\hline Bandwidth(MHz) & $20 / 40 / 80 / 160$ & $20 / 40 / 80$ \\
\hline FFT Length & $64 / 128 / 256 / 512$ & $64 / 128 / 256$ \\
\hline Subcarrier Used & $52-500$ & $52-244$ \\
\hline Subcarrier Spacing & \multicolumn{2}{|c|}{$312.5 \mathrm{KHz}$} \\
\hline FFT Period & \multicolumn{2}{|c|}{$3.2 \mathrm{us}$} \\
\hline GI Period & \multicolumn{2}{|c|}{$0.8 \mathrm{us}$} \\
\hline
\end{tabular}

The packet format of the system is devised as shown in Figure 1. There are 14 symbols in preamble, including 10 short and 4 long preamble. In order to support adaptive modulation, we design a 1-bit EXTEND bit; when set to 1, it informs that there is an extra extended field containing more parameters about the packet. Also, when the system is operating as an MIMO transceiver, Alamouti code [6] is adopted in the long 
preamble pattern transmitted by two antennas to estimate channel frequency response.

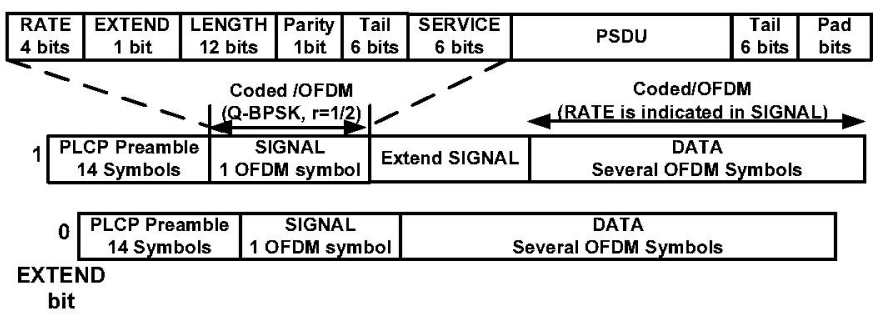

Fig. 1. Packet Format.

\section{B. Channel Model}

The channel model used for simulation is based on IEEE 802.11 TGn standard, which stipulates 6 different MIMO channel models, representing various indoor environments [7]. Based on these MIMO channels, we construct the equivalent baseband channel model by adding multipath Rayleigh fading, additive white gaussian noise (AWGN), carrier frequency offset (CFO) and sampling clock offset (SCO). To speed up the simulation process, we propose an approach that considers the interpolation needed in modeling multipath and SCO effects at the same time, which also gives a closer channel emulation to the real environments.

\section{Parameterized Transceiver Design}

\section{A. Transmitter}

The $2 \times 2$ MIMO-OFDM transmitter block diagram is shown in Figure 2. The binary data stream first passes through constellation mapper according to the modulation types. Afterwards, the data stream is allocated to the corresponding data subcarrier positions and then sent into the IFFT block, where preamble is inserted. Finally, the GI is added to complete the generation of the time-domain transmitted signal. The baseband signal is then up-converted and sent out through the transmitting antennas.

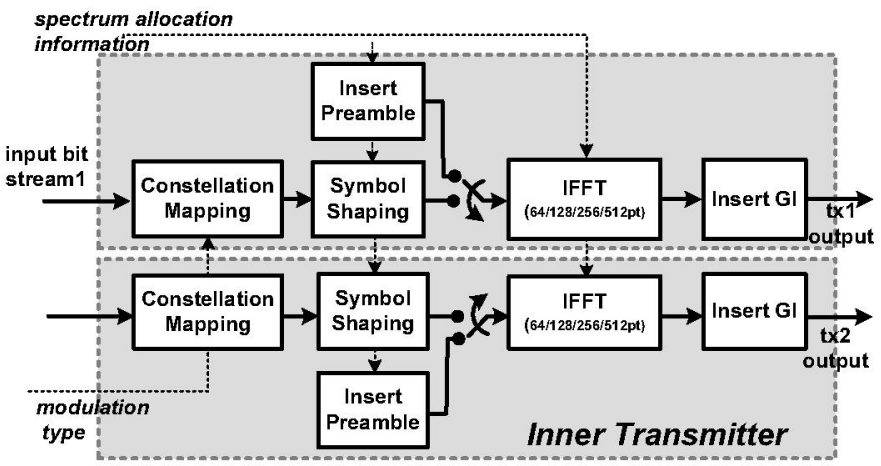

Fig. 2. Block diagram of the baseband CR transmitter.

\section{B. Receiver}

The receiver can be partitioned into three parts: initial synchronization, CFO and SCO tracking loops, channel estimation as well as data recovery, which also represents the operation flow of the receiver. The proposed receiver architecture and initial synchronization flow are depicted in Figures 3 and 4, respectively. Since OFDM system is very sensitive to timing and frequency deviation, the system begins synchronization by computing short preamble (SP) delay correlation to estimate coarse symbol timing and coarse CFO. From Figure 4, with the autocorrelation property of SP, the coarse CFO can be estimated from the peak position of delay correlation in step 2 while the coarse symbol timing can be predicted from the position of the half peak value found in step 3. Similarly, the long preamble (LP), passed into matched filter, offers the mechanism to find fine symbol timing by firstly getting the peak position of matched filter output as in step 5 and trace back in time until seeing a strong enough multipath component to determine the window containing maximum energy as in step 6. Lastly, fine CFO estimation can be performed by observing delay correlation result from LP, depicted in step 7.

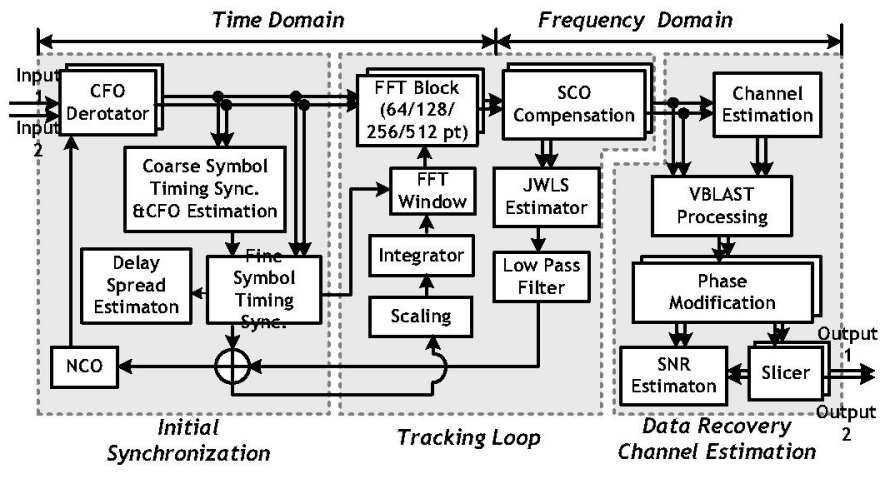

Fig. 3. Block diagram of the baseband CR Receiver.

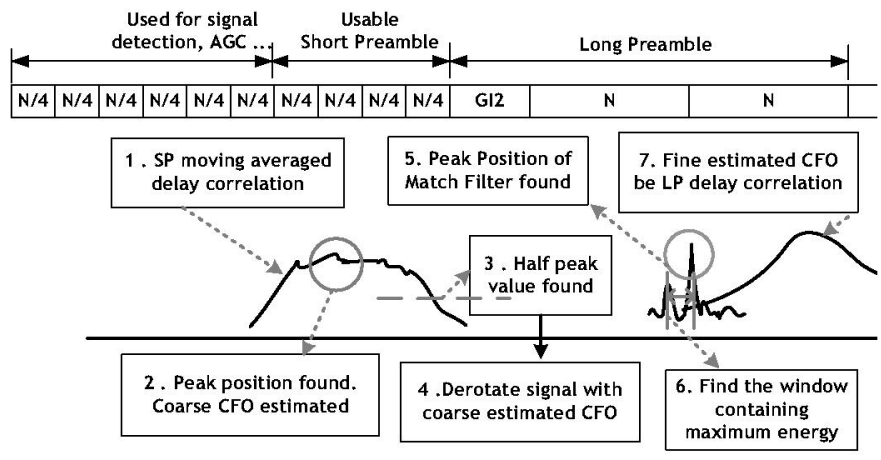

Fig. 4. Operation flow of the proposed receiver.

When the system completes initial synchronization, it then enters into tracking mode. As shown in Figure 3, SCO compensation, JWLS estimator [8], low pass filter, NCO form the feedback path to control CFO derotator. And the scaling block, integrator, FFT window try to set the proper FFT timing 
boundary so as to keep track of the SCO. In the mean time, the channel estimation, phase modification and slicer start to recover the received data. From simulation result, the accuracy of estimated channel frequency response degrades more under lower SNR condition. As channel estimation is pivotal to the entire data recovery flow, we then smooth acquired channel frequency response through raised cosine filter, of which the time domain response is:

$$
x(t)= \begin{cases}1 & \text { if } 0 \leq|t| \leq \frac{1-\beta}{2} T \\ \frac{\left(1+\cos \frac{\pi}{\beta}\left(|t|-\frac{1-\beta}{2} T\right)\right)}{2} & \text { if } \frac{1-\beta}{2} T \leq|t| \leq \frac{1+\beta}{2} T \\ \text { elsewhere. }\end{cases}
$$

Thus, the frequency domain filtering function for smoothing is:

$$
X(f)=\sin c(f T) \frac{\cos (\pi \beta f T)}{1-4 \beta^{2} f^{2} T^{2}},
$$

where $\beta$ is the roll-off factor, and $T$ is the width of the time domain window, the length of which is chosen to be that of guard interval because the maximum interval of statistical delay spread profile is less than the duration of GI.

Lastly, V-BLAST processing for equalization and detection can further enhance data rate and lower bit error rate when MIMO is applied. The linear combinational nulling concept is adopted for the detection of V-BLAST scheme, which is outlined as follows:

Step 1: Determine the optimal detection order of received signal.

Step 2: Suppose the determined detection order is

$$
k_{1}, k_{2}, \ldots
$$

We then pick the first stream as target signal while treat other streams as interference, which are later canceled by using proper weights and vector multiplication so as to get the detected first stream.

Step 3: Subtract all the remaining streams by the detected value we get in Step 2, then return to Step 1, continuing iteration of the above steps until we detect all streams.

\section{Parameterized Design}

To realize a CR system, the proposed transceiver is capable of adjusting the parameters of certain functional blocks as the channel changes its characteristics. Table II lists those receiver functional blocks in which some circuit parameters will be adjusted according to the channel conditions; the major criterion is the FFT size, which can be obtained through the information provided by the sensing block. The capability of dynamically adjusting these parameters indicates the profound reconfigurability of our system, meeting the demand for CR system.

\section{Sensing block}

Another major CR system requirement is the ability to assess the characteristics or quality of the channel. In our system, we realize two functions: SNR estimation and delay spread estimation, as shown in Figure 3. We use short preamble delay
TABLE II

ADAPTIVE SYSTEM PARAMETERS

\begin{tabular}{|c|}
\hline Coarse Symbol Timing Synchronization \\
\hline Matched Filter \\
\hline FFT Block \\
\hline JWLS \\
\hline SCO Compensation \\
\hline Channel Estimation \\
\hline Equalization \\
\hline Phase Modification \\
\hline Slicer \\
\hline
\end{tabular}

correlation result to estimate the SNR value [9]. Meanwhile, matched filter is used to compute the channel impulse response for estimating channel delay spread. Hardware sharing concept is realized by reusing the delay correlation and matched filter circuits, which are also needed during initial synchronization.

\section{Simulation Result}

\section{A. System Verification}

To verify the correctness of the proposed system, we build a functional simulation model for our system to compare our design with theoretical performance. Hence, we set the receiver under SISO mode with perfect channel estimation, timing synchronization and AWGN only channel environment, which enables us to plot packet error rate (PER) to see if the system performance meets the requirements from 802.11a. Figure 5 shows that the PER curves of all four modulations coincide with those obtained from theoretical analysis, indicating competitiveness of the proposed receiver.

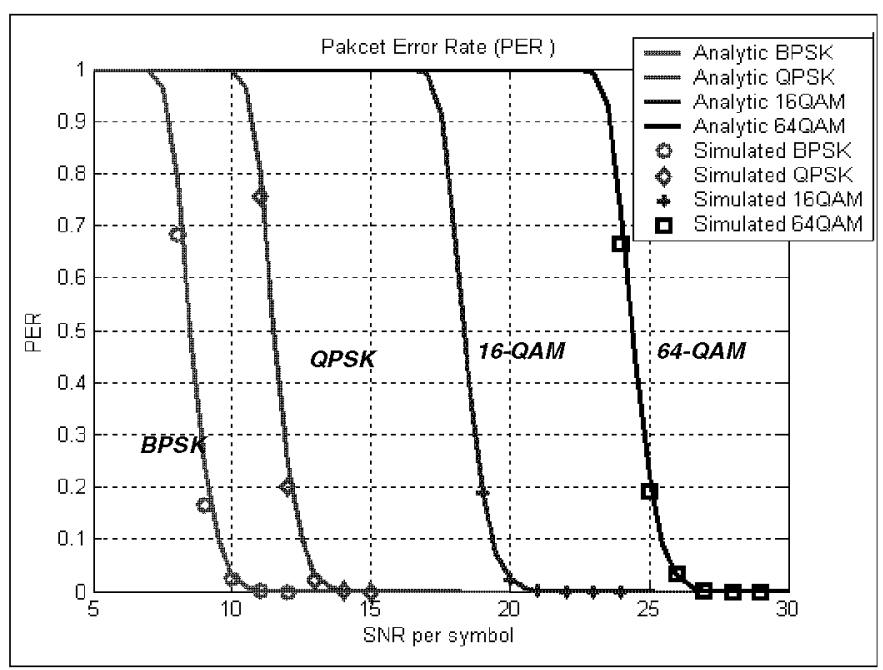

Fig. 5. Packet error rate (PER) comparison.

\section{B. Simulation with/without $C F O$ and $S C O$}

The bit error rate (BER) comparisons between the two cases of no $\mathrm{CFO} / \mathrm{SCO}$ and $\mathrm{CFO} / \mathrm{SCO}$ with compensation are shown in Figure 6. Under different types of channel, we can see the BER performance in the case with $\mathrm{SCO}=13.7 \mathrm{ppm}, \mathrm{CFO}$ 
$=0.254 \cdot \Delta \mathrm{f}$ and compensation for both is very close to the no $\mathrm{CFO} / \mathrm{SCO}$ case. The simulation result using $64 \mathrm{QAM}$ under channel model B, D, and E demonstrates the system's effectiveness in compensating SCO and CFO effects.

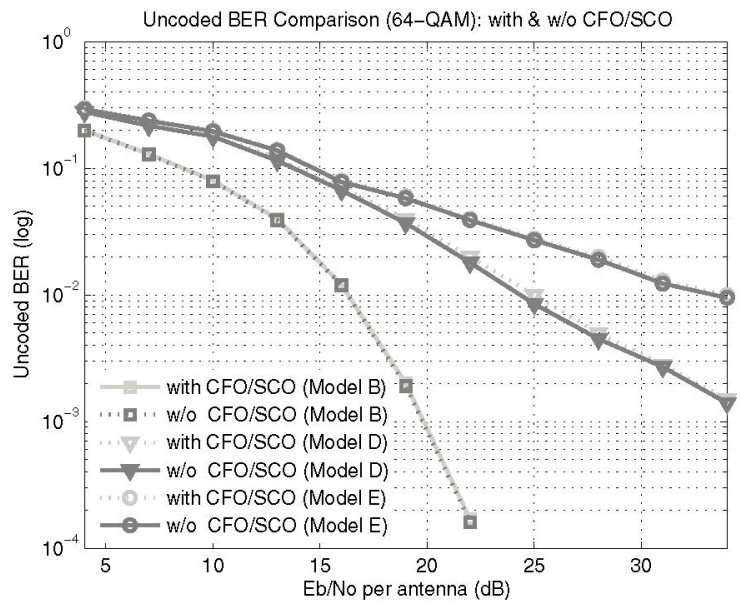

Fig. 6. Comparisons between no $\mathrm{CFO} / \mathrm{SCO}$ case and $\mathrm{CFO} / \mathrm{SCO}$ with compensation case.

\section{Overall System Performance}

To evaluate the overall system performance, we use six different transmission types, three of which shown in Table III, to simulate our inner receiver. Because we use lower UNII band in these simulations, there are up to 8 bands available in transmission as in T6. Variable $\mathrm{U}$ denotes the capability to adjust data rate by using different kinds of modulation. For $\mathrm{U}=6$, the modulation is $64 \mathrm{QAM}$; hence, the maximum data rate in our system is $234 \cdot 6=1404 \mathrm{Mbps}$. For $\mathrm{U}=$ 1,2 , or 4 , the modulation scheme corresponds to BPSK, QPSK, 16QAM. We then plot one simulation result under T1 transmission type with modulation of QPSK in Figure 7. From the simulation result, we see that uncoded BER less than $10^{-3}$ is achieved with $15 \mathrm{~dB}$ SNR under channel type B. Also, under more severe multipath channels, i.e., model D and E, some BER degradation is observed, showing a BER of $10^{-2}$ with about SNR of 20 and $30 \mathrm{~dB}$ under channel model $\mathrm{D}$ and $\mathrm{E}$, respectively.

TABLE III

TRANSMISSION TYPES.

\begin{tabular}{|c|c|c|c|}
\hline Transmission Type & T1 & T4 & T6 \\
\hline Band Allocation & 1 & $1-4$ & $1-8$ \\
\hline Data Rate $(\mathrm{U}=1,2,4,6)$ & $24 \mathrm{U}$ & $114 \mathrm{U}$ & $234 \mathrm{U}$ \\
\hline BW $(\mathrm{MHz})$ & 20 & 80 & 160 \\
\hline CFO & 0.2271 & 0.2284 & 0.2302 \\
\hline
\end{tabular}

\section{Conclusion}

In this paper, we present a baseband transceiver system architecture for CR application. In order not to disturb the primary users in operation, the spectrum allocation of our system needs to be dynamically adjusted. Such arrangement

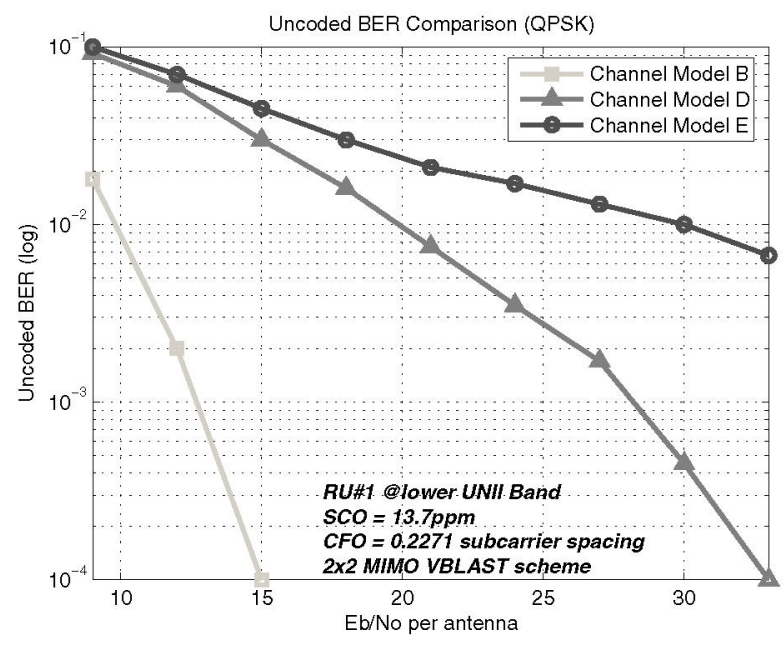

Fig. 7. Bit error rate performance of the proposed receiver.

entails two physical layer design considerations: the parametric design and channel quality assessment. The proposed system uses OFDM as the transmission scheme and also incorporates MIMO technique to enable flexible adjustment of data rate according to the estimated channel characteristics. The proposed receiver integrates synchronization, tracking, and channel estimation mechanisms in accordance with a WLANlike packet. Additionally, the sensing and data transmission abilities as well as BER and PER performance compiled from functional simulation confirm the effectiveness of the proposed transceiver in CR applications.

\section{ACKNOWLEDGMENT}

The authors would like to thank MediaTek Inc. for supporting this work.

\section{REFERENCES}

[1] FCC., ET Docket No 02-155, "Spectrum Policy Task Force Report," Order, Nov. 2002.

[2] T. A. Weiss, F. K. Jondral, "Spectrum Pooling: An Innovative Strategy for the Enhancement of Spectrum Efficiency," Vol. 42, pp. 8-14, Mar. 2004.

[3] FCC. ET Docket No. 03-322, "Notice of Proposed Rule Making and Order," Dec. 2003.

[4] Danijela Cabric, "Research Opportunities in Cognitive Radios," in BWRC Winter Retreat, Jan. 2004

[5] P. M. Wolniansky, G. J. Foschini, G. D. Golden, R. A. Valenzuela, “VBLAST: An Architecture For Realizing Very High Data Rates Over the Rich-Scattering Wireless Channel," International Symposium on Signals, Systems, and Electronics, pp. 295-300, Sep. 1998.

[6] S. M. Alamouti, "A Simple Transmit Diversity Technique for Wireless Communications," IEEE J. of Selected Areas in Comm., pp. 1451-1458, 1998.

[7] IEEE 802.11-03/9404r4 TGn Channel Models.

[8] P. Y. Tsai, H. Y. Kang and T. D. Chiueh, "Joint Weighted Least Squares Estimation of Carrier Frequency and Timing Frequency Offset for OFDM Systems over Multipath Fading Channel," IEEE Trans. on Vehicular Technology, vol. 54, No.1, pp. 211-223, January 2005.

[9] T. M. Schmidl, D. C. Cox, "Robust Frequency and Timing Synchronization for OFDM," IEEE Trans. on Comm., Vol. 45, pp. 1613-1621, Dec. 1997. 